\title{
Programming colloidal phase transitions with DNA strand displacement
}

\section{Citation}

Rogers, W. B., and V. N. Manoharan. 2015. “Programming Colloidal Phase Transitions with DNA Strand Displacement.” Science 347 (6222) (February 5): 639-642. doi:10.1126/science.1259762.

\section{Published Version}

doi:10.1126/science.1259762

\section{Permanent link}

http://nrs.harvard.edu/urn-3:HUL.InstRepos:24902852

\section{Terms of Use}

This article was downloaded from Harvard University's DASH repository, and is made available under the terms and conditions applicable to Other Posted Material, as set forth at http:// nrs.harvard.edu/urn-3:HUL.InstRepos:dash.current.terms-of-use\#LAA

\section{Share Your Story}

The Harvard community has made this article openly available.

Please share how this access benefits you. Submit a story.

\section{Accessibility}




\title{
Programming colloidal phase transitions with DNA strand displacement*
}

\author{
W. Benjamin Rogers ${ }^{1}$ and Vinothan N. Manoharan ${ }^{\dagger 1,2}$ \\ ${ }^{1}$ School of Engineering and Applied Sciences, Harvard University, Cambridge MA \\ ${ }^{2}$ Department of Physics, Harvard University, Cambridge MA 02138 USA
}

One sentence summary: DNA sequences can be used to finely control the temperature-dependent phase behavior of DNA-connected colloidal particles.

DNA-grafted nanoparticles have been called "programmable atomequivalents": Like atoms, they form three-dimensional crystals, but unlike atoms, the particles themselves carry information (the sequences of the grafted strands) that can be used to "program" the equilibrium crystal structures. We show that the programmability of these colloids can be generalized to the full temperaturedependent phase diagram, and not just the crystal structures. We add information to the buffer, in the form of soluble DNA strands designed to compete with the grafted strands through strand displacement. Employing only two displacement reactions, we program phase behavior not found in atomic systems or other DNAgrafted colloids, including arbitrarily wide gas-solid coexistence, re-entrant melting, and even reversible transitions between distinct crystal phases.

\footnotetext{
*This is the authors' version of the work. It is posted here by permission of the AAAS for personal use, not for redistribution. The definitive version was published in Science volume 347 on February 6, 2015, DOI: 10.1126/science.1259762

†To whom correspondence should be addressed. E-mail: vnm@seas.harvard.edu
} 
Like atoms, colloidal particles suspended in a fluid can form bulk phases such as gases and crystals. Such particles can also be directed to form new states of matter (1) through careful tuning of their interparticle interactions, for example by grafting DNA strands onto the particles to create specific attractions $(2,3)$. Such DNA-grafted particles have been called "programmable atom-equivalents" (4), a moniker that highlights the experimenter's ability to dictate, or "program," the selfassembled structures through the DNA sequences. The implied analogy to computer programming is a useful way to conceptualize how information in the sequences is translated to structure: Much as one can program a computer to perform complex tasks by writing statements that are compiled to machine code, one can "program" a colloid to form a complex structure by designing nucleotide sequences (statements) that are "compiled" into specific interparticle interactions (machine code). Recent advances in our understanding of this compilation process, in the form of design rules $(5)$ or mean-field models $(6,7,8)$ relating the effective interactions directly to the nucleotide sequences (9), have enabled the assembly of crystal phases not found in ordinary colloids $(10,11,5,12,13)$ and could be extended, in principle, to assembly of prescribed, nonperiodic structures $(14,15)$.

Structure, however, is just one aspect of self-assembly; more generally, selfassembly describes a phase transition between a disordered and ordered state, or a pathway on a phase diagram. Thus far only a subset of the full colloidal phase diagram has been programmed: the equilibrium structure of the ordered state as a function of density and composition. Programmatic control over the phase behavior in the orthogonal thermodynamic dimension, the temperature, remains elusive. Typically, the attraction between two DNA-grafted particles decreases steeply and monotonically with increasing temperature $(16,17)$. As a result, the suspension is fluid at high temperature and solid at low temperature-phase behavior resembling that of simple atoms, rather than programmable ones (Fig. 1A). Our goal here is to develop a comprehensive approach to programming the full phase diagram of colloidal suspensions: we seek to design a set of interaction "primitives" that can be combined to program both the structure of equilibrium phases and their temperature-dependent transitions. In other words, we aim to program the equilibrium self-assembly pathways, and not just their end points.

We achieve this goal by adding information to the buffer in the form of free DNA strands. We call these strands displacing strands since their sequences are designed to be complementary to subunits of the grafted strands; they can therefore react with a double-stranded bridge, displacing one of the grafted strands and forming a non-bridging duplex (Fig. 1B). This hybridization reaction, known as toehold exchange or strand displacement, is widely used in the DNA nanotechnology field to construct dynamic assemblies and devices $(18,19)$. Strand displacement has also been used to melt or change the lattice constants of nanoparticle-based materials $(20,21,22,23)$. Here, rather than modifying the structure of an already as- 
W. B. Rogers, V. N. Manoharan, Science 347: 639 (2015)

A

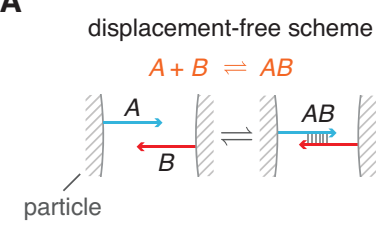

B

B one-displacement scheme

$A+B \rightleftharpoons A B$

$A B+D_{1} \rightleftharpoons A D_{1}+B$

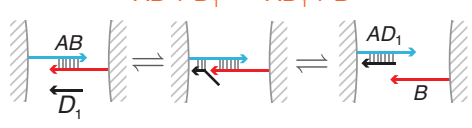

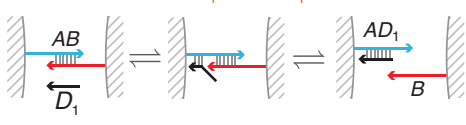

C

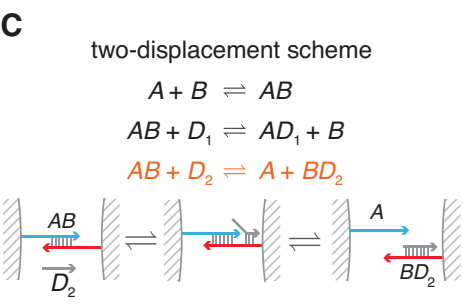

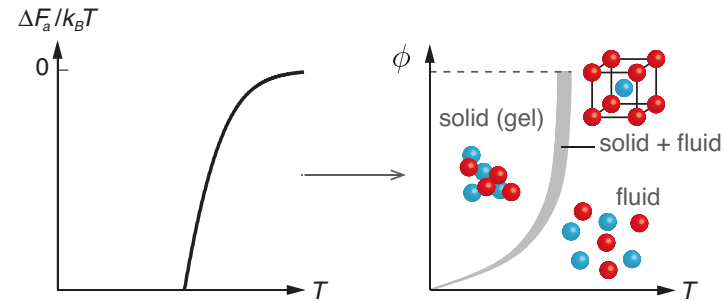
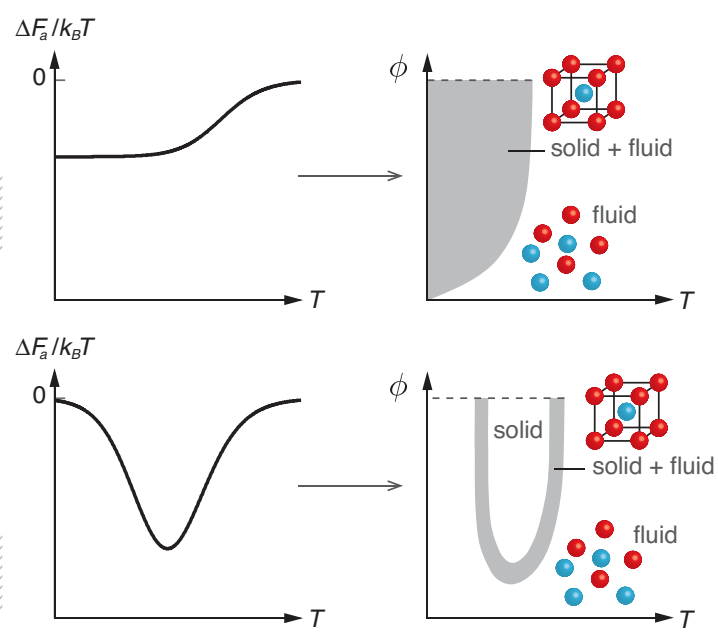

Figure 1: Strand-displacement reactions program phase behavior by modifying the local chemical equilibrium between DNA-grafted particles. (A) In the absence of displacing strands, the strength of the DNA-induced attraction $\left(\Delta F_{a}\right)$ decreases monotonically with increasing temperature $T$, resulting in simple phase behavior in the $\phi-T$ space, where $\phi$ is the particle volume fraction. The fluid-solid coexistence region is shown in gray. (B) A single displacement reaction eliminates the temperature dependence of $\Delta F_{a}$ over a range of temperatures, dramatically widening the fluid-solid coexistence region. (C) Adding a second strand-displacement reaction allows $\Delta F_{a}$ to vary non-monotonically with $T$, inverting the colloidal phase behavior and creating a re-entrant fluid phase. The elementary reaction steps in orange are drawn schematically. 
sembled material, we use strand-displacement reactions to control the equilibrium assembly process. The additional degrees of freedom that we introduce allow us to design temperature-dependent interaction potentials with tunable shape, steepness, and specificity (Fig. 1B-C). Returning to the computer programming analogy, the free DNA sequences act as the language for programming the transitions between phases, much as the grafted sequences program the structure of the phases. Because we separate the functions of the grafted and free strands, the two mechanisms can be controlled independently.

To understand how displacing strands affect the interparticle potential, consider the hybridization reactions shown in Fig. 1. Given that hybridization of complementary strands happens on timescales much shorter than that of particle motion, we can assume that interacting DNA strands are in chemical equilibrium $(6,7,8)$. More precisely, the DNA-induced colloidal attraction is determined by the spatially varying hybridization yield of DNA bridges, whose temperature dependence comes from the free energy change $\Delta G / R T$ (see references $(7,24)$ and the Supplementary Materials (25) for more detail on this model). In the absence of displacement, the free energy change of the hybridization reaction $A+B \leftrightharpoons A B$, given by $\Delta G / R T=\Delta H_{A B} / R T-\Delta S_{A B} / R$, is monotonic with a steepness set by $\Delta H_{A B}$, since the enthalpy change $\Delta H_{A B}$ and entropy change $\Delta S_{A B}$ are largely independent of temperature (Fig. 1A).

With displacing strands, the free energy difference between bridged and unbridged states can be modified owing to the additional reaction pathways

$$
\begin{aligned}
& A B+D_{1} \leftrightharpoons A D_{1}+B \\
& A B+D_{2} \leftrightharpoons A+B D_{2} .
\end{aligned}
$$

Because the enthalpic changes of displacement reactions can be tuned through the base sequences of the displacing strands, the free energy change $\Delta G^{\prime} / R T$ can be designed to have various nonlinear dependences on temperature (Figs. S1 and S2). Furthermore, the entropic changes of the displacement reactions can also be adjusted by changing the molar concentrations of the displacing strands, providing a way to tune the magnitude of $\Delta G^{\prime} / R T$ independently of its dependence on temperature.

A single displacement reaction (Fig. 2A) allows precise control over the thermodynamics of the fluid-solid transition. We control the temperature dependence of the free energy change $\Delta G^{\prime} / R T$, and thus of the interaction potential, by changing the displacing strand sequence. Using the nearest-neighbor model, which relates DNA sequences to hybridization free energies (9), we predict the enthalpic changes of displacement and bridge formation. If we choose the appropriate sequences such that these enthalpic changes are the same $\left(\Delta H_{A B}=\Delta H_{A D_{1}}\right)$, we can eliminate the temperature dependence entirely over a range of temperatures (Fig. S1). We thereby 
W. B. Rogers, V. N. Manoharan, Science 347: 639 (2015)

A
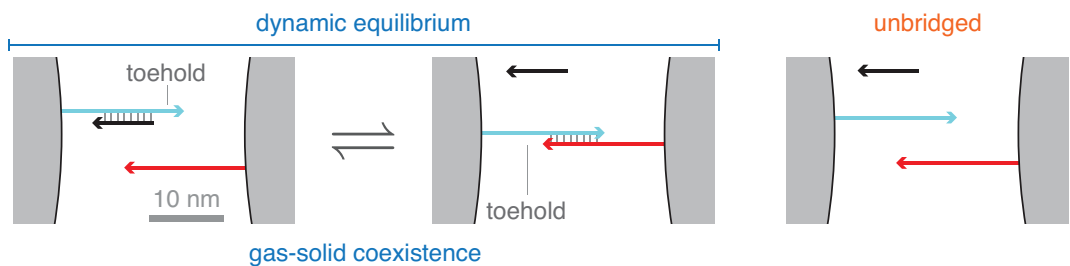

B

gas-solid coexistence
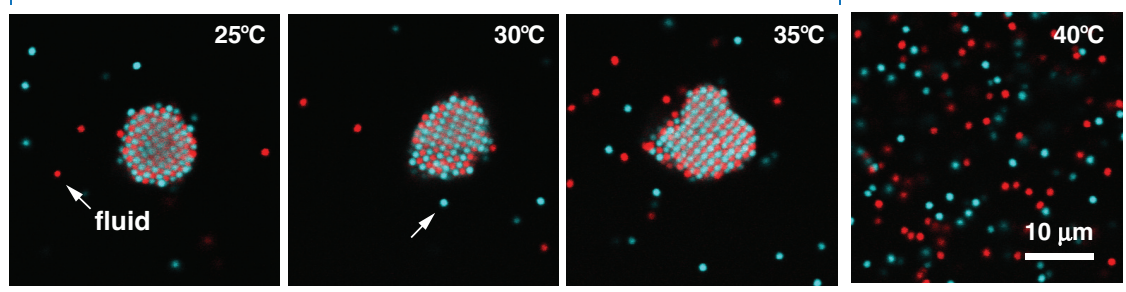

C

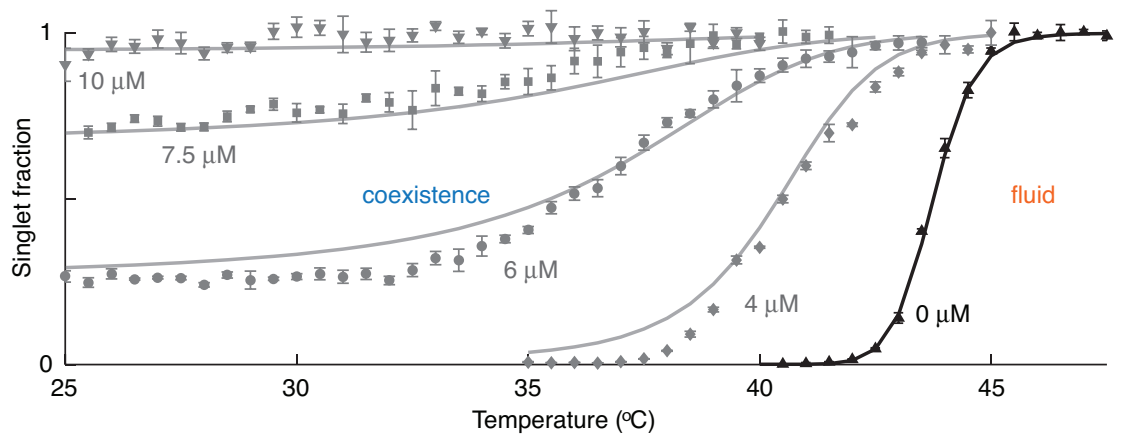

Figure 2: A single displacement reaction eliminates the temperature dependence of binding. (A) Competition between bridge formation and strand displacement results in stable coexistence between fluid and solid phases that persists over a wide range of temperatures, as seen by confocal microscopy (B). (C) Experimentally-measured particle singlet fraction (symbols) shows the broadening of the melting transition with increasing concentration of free strand $D_{1}$ (indicated on plot) (25). Error bars are the standard deviation of three measurements. A model based on local chemical equilibrium (curves), together with a separate model of the singlet fraction (16), reproduces our results to within the inherent uncertainty of the nearest-neighbor model $(25,9,32)$. DNA sequences and predicted free energies are given in Table S1 and Table $\mathrm{S} 2$. 
establish a dynamic equilibrium in which the bridging and non-bridging duplexes exchange freely by toehold-exchange hybridization, without an enthalpic barrier.

This single-displacement scheme, where $\Delta H_{A B}=\Delta H_{A D_{1}}$, eliminates the boundary between the coexistence region and the solid phase, resulting in coexistence between fluid and solid that persists even at low temperatures (Fig. 2B). In the absence of the displacing strand, we find a single, steep melting curve with an approximate width of $1{ }^{\circ} \mathrm{C}$, consistent with earlier reports (16). The melting transition softens with increasing concentration of the free strand (Fig. 2C), widening by $10^{\circ} \mathrm{C}$ or more. Furthermore, the singlet fraction remains nonzero and constant down to room temperature. Because the entropy of the free strands can be adjusted by changing their molar concentration, the singlet fraction, and thus the interaction strength, can still be tuned.

This single-displacement scheme solves a longstanding problem in DNA-directed self-assembly: the steep dependence of the interparticle attraction on temperature (17), which frustrates equilibrium self-assembly. Previous experiments and simulations have shown that crystal nucleation and growth occur over a range of interaction strengths only 1-2 $k_{B} T$ wide, corresponding to a temperature window roughly $1{ }^{\circ} \mathrm{C}$ wide $(6,26)$. In contrast, with a single displacement reaction, we find that nucleation and growth of binary crystals occurs over a range of temperatures wider than $10{ }^{\circ} \mathrm{C}$, at least an order-of-magnitude improvement compared to displacement-free schemes. Expanding the temperature window of equilibrium assembly makes it easier to grow crystals and obviates the need for precision temperature control, temperature gradients, or complex annealing schemes $(10,11,13)$.

Our model of DNA-mediated attractions in the presence of strand displacement quantitatively reproduces these measurements (Fig. 2C). Taking the grafting density, free strand concentration, ionic strength, and DNA sequences as inputs, we reproduce the measured singlet fractions to within the inherent uncertainty associated with the nearest-neighbor model (25). This level of agreement supports our physical picture-that the changes in the temperature dependence result directly from molecular scale displacement reactions-and demonstrates that the emergent phase behavior can be predicted, and therefore programmed.

With two displacement reactions (Fig. 3A), we can make the free energy not only a nonlinear function of temperature but also a non-monotonic one, with interesting consequences for the phase behavior: The resulting suspensions display multiple fluid-solid transitions and inverted phase behavior, in which the stable, low-temperature phase is a fluid that freezes upon heating before melting again at higher temperatures. Such re-entrant behavior results from a competition between entropy and enthalpy. The low-temperature fluid is stabilized enthalpically: because each bridge can be replaced by two non-bridging duplexes of the same length, the most favorable state contains few or no bridges between particles, thus maximizing the total number of base pairs. At higher temperatures, entropy favors the solid 
phase, since formation of a single bridge liberates two displacing strands. At even higher temperatures, the solid phase melts again, owing to thermal dissociation of DNA bridges.

Our experiments (Fig. 3B) show that the resulting re-entrant melting transition is tunable and can be programmed independently of the solid phase symmetry. By adjusting the concentration of the displacing strands, we control the temperature window in which the solid phase is stable (Fig. 3C). Higher concentrations of displacing strands shift the local chemical equilibrium toward non-bridging duplexes, leading to a narrower window (Fig. 3D). Strand concentrations exceeding a critical limit prevent freezing entirely. The crystals that we assemble have the expected cesium chloride $(\mathrm{CsCl})$ symmetry (Fig. S3). Because energetic arguments suggest that intraspecies attractions as weak as roughly $1 k_{B} T$ would lead to formation of $\mathrm{Cu}$-Au crystals instead of the observed $\mathrm{CsCl}$ crystals $(13,27)$, we conclude that our approach does not result in undesired crosstalk between intra- and interspecies attractions.

Of course, the principal feature of DNA-grafted particles is the ability to create multiple particle species that interact with each other in specific ways. Strand displacement allows us to modify each interaction and thereby program pathways between different self-assembled structures. To demonstrate this feature of our approach, we combine the displacement-free and two-displacement schemes to program a reversible pathway between two equilibrium, compositionally-distinct ordered phases. Specifically, we design a system containing three particle species with a temperature-dependent interaction matrix, implemented through six DNA sequences (Table S5), four of which are grafted to particles and two of which are displacing strands that modulate interactions between species 2 (green in Fig. 4A) and the other two species. At low temperatures, the interaction matrix favors cocrystallization of species 2 and 3, as confirmed by confocal fluorescence microscopy (Fig. 4B). At high temperatures, it favors co-crystallization of species 1 and 2 . At intermediate temperatures, we program an intervening fluid phase by tuning the displacing strand concentrations, which allows us to easily nucleate and grow either crystal by lowering or raising the temperature. Because the system is in equilibrium at each temperature, the observed phase transitions are completely reversible.

These last experiments demonstrate that specificity afforded by Watson-Crick base pairing, which is used to program the structure of equilibrium self-assembled phases, can itself be programmed to depend on temperature, enabling reconfigurable materials in which particles change their interactions and reconfigure their structure in response to temperature. The approach is limited only by the freezing and boiling points of the buffer: because the transition illustrated in Fig. 4A-B is roughly $10{ }^{\circ} \mathrm{C}$ wide, one could conceivably design transitions between at least ten distinct solid phases in the $0-100{ }^{\circ} \mathrm{C}$ temperature range, which could each be directed to self-assemble independently and on cue simply by changing the temperature. More- 
W. B. Rogers, V. N. Manoharan, Science 347: 639 (2015)

A

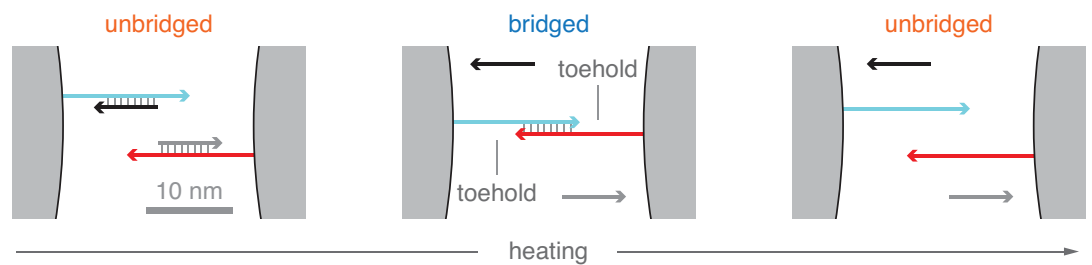

B
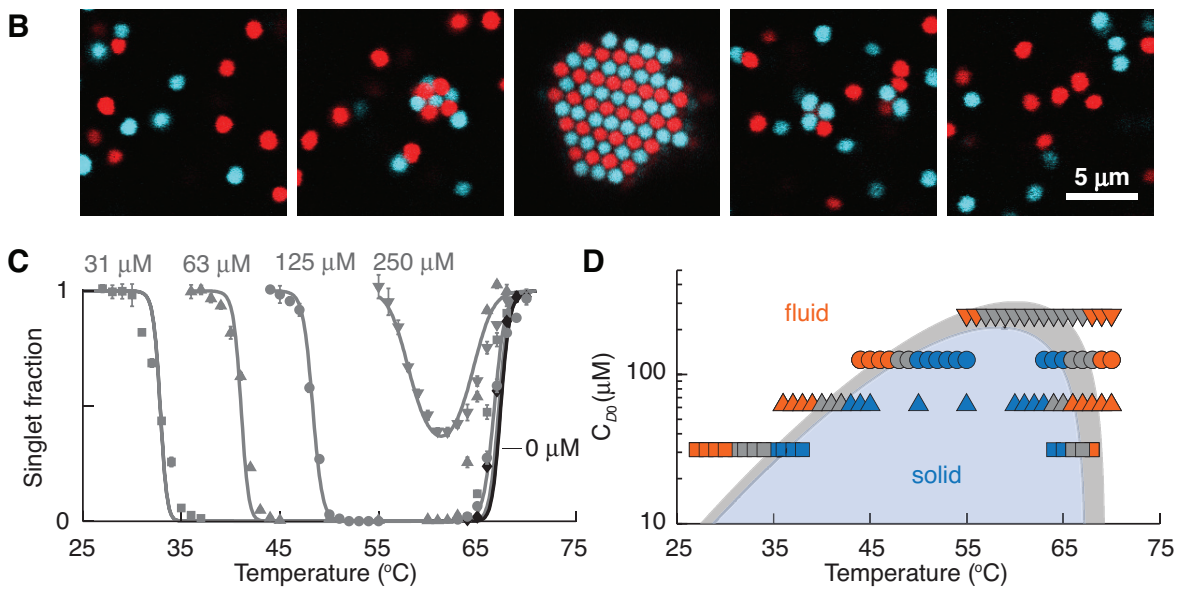

Figure 3: Two strand-displacement reactions program a tunable re-entrant melting transition. (A) Hybridization of free displacing strands induces a second melting transition, as seen by confocal microscopy (B). (C) Singlet fraction $f$ measurements (symbols) show that the re-entrant melting transition can be tuned by changing the displacing strand concentrations $C_{D 0}$ for equimolar mixtures of $D_{1}$ and $D_{2}$ (indicated on plot) (25). Error bars are the standard deviation of three measurements. Our local chemical equilibrium model (curves) reproduces our results to within the inherent uncertainty of nearest-neighbor predictions $(25,9,32)$. (D) The displacing strand concentration-temperature coexistence envelope is delimited by the temperature and $C_{D 0}$ where $0.15<f<0.85$ (gray). Symbols show experimental data: orange for $f>0.85$, blue for $f<0.15$. We achieve coexistence over roughly $10{ }^{\circ} \mathrm{C}$ when $C_{D 0}=250 \mu \mathrm{M}$. DNA sequences and hybridization free energies are shown in Tables S3 and S4. 


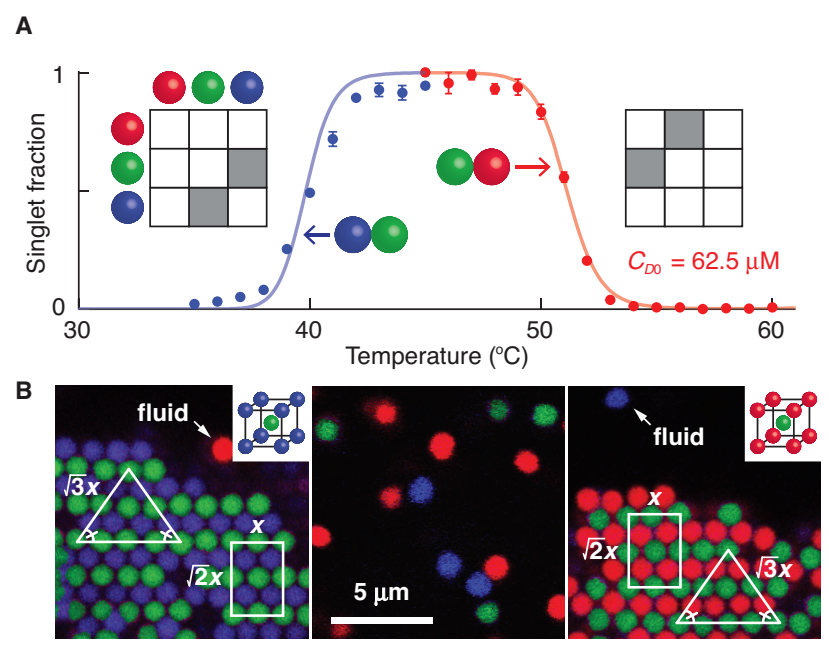

Figure 4: The zero- and two-displacement reaction schemes are combined to program a pathway between two colloidal crystals. (A) Strand displacement yields a temperature-dependent specificity matrix defining favorable (gray) and unfavorable (white) interactions in a ternary suspension. Measured pair interactions (symbols) in this experimental system agree quantitatively with our model calculations (curves). Error bars are the standard deviation of three measurements. (B) Confocal fluorescence experiments (25) show $\mathrm{CsCl}$ binary crystals of species 2 (green) and 3 (blue) in coexistence with a fluid of species 1 (red) at low temperature (left), and $\mathrm{CsCl}$ crystals of species 1 (red) and 2 (green) in coexistence with a fluid of species 3 (blue) at high temperature (right), separated by a homogeneous fluid phase of all three species at intermediate temperature (middle), as predicted. The two crystals have same symmetry, as determined by the lattice distance $x=\frac{4}{\sqrt{3}} D$ in the $\{110\}$ plane, but different compositions; $D$ is the particle diameter. Hybridization free energies are shown in Table S6.

over, incorporation of thermally driven solid-solid transitions could also enable the sequential self-assembly of other crystal phases not accessible by direct nucleation from the fluid, but which have the lowest free energy at a given temperature (13). These systems represent an additional direction in self-assembly, in which information supplied to the buffer can program equilibrium pathways between many different target structures within a closed system.

The zero-, one-, and two-displacement reaction schemes constitute a set of primitives that can be combined to further program thermal pathways to self-assembly. We have demonstrated one such combination-a zero-displacement reaction combined with a two-displacement reaction-but many others are possible, owing to the specificity of DNA hybridization. A key feature of our approach is that it separates the functions of the grafted strands, which encode the interaction matrix, and the 
free displacing strands, which control the temperature dependence of the interaction matrix. Other competitive binding schemes have been proposed $(28,29,30)$, but none result in independent control of the temperature-dependent phase transitions and the symmetry of the equilibrium phases. This independent control, which is crucial to fully program self-assembly, could make it possible to assemble complex materials in multiple stages. For example, particles might first self-assemble into a scaffold that would disassemble after helping the final, prescribed structure to assemble. Similar strategies are employed in biological systems such as bacteriophages (31) and could prove to be more robust than current one-step assembly approaches. More generally, our demonstration that strand displacement alters the local chemical equilibrium between DNA-grafted particles opens the door to the inclusion of more complex strand displacement-based devices into colloidal assembly. For example, incorporation of DNA-based logic gates, cascaded circuits, or catalytic amplifiers (19) could make it possible to program non-equilibrium selfassembly pathways in colloidal matter.

\section{References and Notes}

1. D. Frenkel, Playing tricks with designer "atoms", Science 296, 65 (2002).

2. C. A. Mirkin, R. L. Letsinger, R. C. Mucic, J. J. Storhoff, A DNA-based method for rationally assembling nanoparticles into macroscopic materials, Nature $\mathbf{3 8 2}$, 607 (1996).

3. A. P. Alivisatos, K. P. Johnsson, X. Peng, T. E. Wilson, C. J. Loweth, M. P. Bruchez, P. G. Schultz, Organization of 'nanocrystal molecules' using DNA, Nature 382, 609 (1996).

4. R. J. Macfarlane, M. N. O'Brien, S. H. Petrosko, C. A. Mirkin, Nucleic acidmodified nanostructures as programmable atom equivalents: Forging a new "table of elements", Angew. Chem. Int. Ed. 52, 5688 (2013).

5. R. Macfarlane, B. Lee, M. Jones, N. Harris, G. Schatz, C. Mirkin, Nanoparticle superlattice engineering with DNA, Science 334, 204 (2011).

6. P. L. Biancaniello, A. J. Kim, J. C. Crocker, Colloidal interactions and selfassembly using DNA hybridization, Phys. Rev. Lett. 94, 058302 (2005).

7. W. B. Rogers, J. C. Crocker, Direct measurements of DNA-mediated colloidal interactions and their quantitative modeling, Proc. Natl Acad. Sci. USA 108, 15687 (2011). 
W. B. Rogers, V. N. Manoharan, Science 347: 639 (2015)

8. P. Varilly, S. Angioletti-Uberti, B. Mognetti, D. Frenkel, A general theory of DNA-mediated and other valence-limited colloidal interactions, J. Chem. Phys. 137, 094108 (2012).

9. J. SantaLucia, A unified view of polymer, dumbbell, and oligonucleotide DNA nearest-neighbor thermodynamics, Proc. Natl Acad. Sci. USA 95, 1460 (1998).

10. D. Nykypanchuk, M. Maye, D. van der Lelie, O. Gang, DNA-guided crystallization of colloidal nanoparticles, Nature 451, 549 (2008).

11. S. Park, A. Lytton-Jean, B. Lee, S. Weigand, G. Schatz, C. Mirkin, DNAprogrammable nanoparticle crystallization, Nature 451, 553 (2008).

12. R. J. Macfarlane, M. R. Jones, B. Lee, E. Auyeung, C. A. Mirkin, Topotactic interconversion of nanoparticle superlattices, Science 341, 1222 (2013).

13. M. Casey, R. Scarlett, W. Rogers, I. Jenkins, T. Sinno, J. Crocker, Driving diffusionless transformations in colloidal crystals using DNA handshaking, Nat. Commun. 3, 1209 (2012).

14. J. D. Halverson, A. V. Tkachenko, DNA-programmed mesoscopic architecture, Phys. Rev. E 87, 062310 (2013).

15. Z. Zeravcic, V. N. Manoharan, M. P. Brenner, Size limits of self-assembled colloidal structures made using specific interactions, Proc. Natl Acad. Sci. USA 111, 15918 (2014).

16. R. Dreyfus, M. E. Leunissen, R. Sha, A. V. Tkachenko, N. C. Seeman, D. J. Pine, P. M. Chaikin, Simple quantitative model for the reversible association of DNA coated colloids, Phys. Rev. Lett. 102, 048301 (2009).

17. L. Di Michele, E. Eiser, Developments in understanding and controlling self assembly of DNA-functionalized colloids, Phys. Chem. Chem. Phys. 15, 3115 (2013).

18. B. Yurke, A. J. Turberfield, A. P. Mills, F. C. Simmel, J. L. Neumann, A DNAfuelled molecular machine made of DNA, Nature 406, 605 (2000).

19. D. Y. Zhang, G. Seelig, Dynamic DNA nanotechnology using stranddisplacement reactions, Nature Chem. 3, 103 (2011).

20. C. K. Tison, V. T. Milam, Reversing DNA-mediated adhesion at a fixed temperature, Langmuir 23, 9728 (2007). 
W. B. Rogers, V. N. Manoharan, Science 347: 639 (2015)

21. M. Maye, M. Kumara, D. Nykypanchuk, W. Sherman, O. Gang, Switching binary states of nanoparticle superlattices and dimer clusters by DNA strands, Nature Nanotech. 5, 116 (2010).

22. J. T. McGinley, I. Jenkins, T. Sinno, J. C. Crocker, Assembling colloidal clusters using crystalline templates and reprogrammable DNA interactions, Soft Matter 9, 9119 (2013).

23. B. A. Baker, G. Mahmoudabadi, V. T. Milam, Strand displacement in DNAbased materials systems, Soft Matter 9, 11160 (2013).

24. W. B. Rogers, J. C. Crocker, Reply to Mognetti et al.: DNA handshaking interaction data are well described by mean-field and molecular models, Proc. Natl Acad. Sci. USA 109, E380 (2012).

25. Materials and methods are available as supplementary material on Science online.

26. T. I. Li, R. Sknepnek, M. Olvera de la Cruz, Thermally active hybridization drives the crystallization of DNA-functionalized nanoparticles, J. Am. Chem. Soc. 135, 8535 (2013).

27. R. Scarlett, M. Ung, J. Crocker, T. Sinno, A mechanistic view of binary colloidal superlattice formation using DNA-directed interactions, Soft Matter 7, 1912 (2011).

28. S. Angioletti-Uberti, B. M. Mognetti, D. Frenkel, Re-entrant melting as a design principle for DNA-coated colloids, Nature Mater. 11, 518 (2012).

29. B. Mognetti, M. Leunissen, D. Frenkel, Controlling the temperature sensitivity of DNA-mediated colloidal interactions through competing linkages, Soft Matter 8, 2213 (2012).

30. S. Roldan-Vargas, F. Smallenburg, W. Kob, F. Sciortino, Gelling by heating, Sci. Rep. 3, 2451 (2013).

31. J. King, S. Casjens, Catalytic head assembling protein in virus morphogenesis, Nature 251, 112 (1974).

32. J. N. Zadeh, C. D. Steenberg, J. S. Bois, B. R. Wolfe, M. B. Pierce, A. R. Khan, R. M. Dirks, N. A. Pierce, Nupack: analysis and design of nucleic acid systems, J. Comput. Chem. 32, 170 (2011).

33. A. Kim, V. Manoharan, J. Crocker, Swelling-based method for preparing stable, functionalized polymer colloids, J. Am. Chem. Soc. 127, 1592 (2005). 
W. B. Rogers, V. N. Manoharan, Science 347: 639 (2015)

34. J. Crocker, D. Grier, et al., Methods of digital video microscopy for colloidal studies, J. Colloid Interface Sci. 179, 298 (1996).

35. K. Wu, L. Feng, R. Sha, R. Dreyfus, A. Grosberg, N. Seeman, P. Chaikin, Polygamous particles, Proceedings of the National Academy of Sciences 109, 18731 (2012).

36. J. SantaLucia, H. T. Allawi, P. A. Seneviratne, Improved nearest-neighbor parameters for predicting DNA duplex stability, Biochemistry 35, 3555 (1996).

37. J. SantaLucia Jr, The use of spectroscopic techniques in the study of DNA stability, Spectrophotometry and Spectrofluorimetry: A Practical Approach pp. 329-56 (2000).

38. B. M. Mognetti, P. Varilly, S. Angioletti-Uberti, F. J. Martinez-Veracoechea, J. Dobnikar, M. E. Leunissen, D. Frenkel, Predicting DNA-mediated colloidal pair interactions, Proc. Natl Acad. Sci. USA 109, E378 (2012).

39. M. Murphy, I. Rasnik, W. Cheng, T. M. Lohman, T. Ha, Probing single-stranded DNA conformational flexibility using fluorescence spectroscopy, Biophys. $J$. 86, 2530 (2004).

40. D. Y. Zhang, S. X. Chen, P. Yin, Optimizing the specificity of nucleic acid hybridization, Nature Chem. 4, 208 (2012).

41. We thank Sofia Magkiriadou, Jesse Collins, Zorana Zeravcic, and Michael Brenner for helpful discussions. We acknowledge support from the Harvard MRSEC, supported by the National Science Foundation under grant number DMR0820484, and from an Alfred P. Sloan Research Fellowship. See Supplementary Materials for additional data.

Supporting Online Material

www.sciencemag.org

Materials and methods

Figs. S1 to S3

References (33)-(40)

Tables S1 to S6. 


\title{
Supplementary Materials for
}

\section{Programming colloidal phase transitions with DNA strand displacement}

\author{
W. Benjamin Rogers, ${ }^{1}$ Vinothan N. Manoharan ${ }^{1,2 *}$ \\ ${ }^{1}$ School of Engineering and Applied Sciences, Harvard University, Cambridge MA 02138 USA \\ ${ }^{2}$ Department of Physics, Harvard University, Cambridge MA 02138 USA \\ *To whom correspondence should be addressed; E-mail: vnm@ seas.harvard.edu
}

\section{This PDF file includes:}

Materials and methods

Figs. S1 to S3

Tables $\mathrm{S} 1$ to $\mathrm{S} 6$

Full reference list 


\section{Materials and methods}

\section{Particle functionalization}

We synthesize DNA-grafted colloidal particles using a technique that physically grafts DNA-conjugated block copolymers to the surface of 1-micrometer-diameter polystyrene microspheres (33). Briefly, the terminal hydroxyl groups of a poly(ethylene oxide)-poly(propylene oxide)-poly(ethylene oxide) triblock copolymer (Pluronic F108; BASF) are activated by $p$-nitrophenyl chloroformate (Sigma-Aldrich). A subsequent reaction with 5'-amino-C6-modified single-stranded DNA (ssDNA) oligonucleotides (Integrated DNA Technologies, Inc.) forms a stable carbamate linkage between F108 and ssDNA. The DNA-conjugated copolymers are then adsorbed to the surface of polystyrene microspheres (Invitrogen) in $10 \mathrm{mM}$ citric acid buffer $(\mathrm{pH}=4)$, and physically grafted by swelling and deswelling the polystyrene cores with toluene. Finally, DNA-grafted particles are washed and stored in an aqueous buffer containing $10 \mathrm{mM}$ Tris and $1 \mathrm{mM}$ EDTA $(\mathrm{pH}=8)$. Typical DNA densities range from $10^{3}-10^{4}$ strands per particle, depending on the starting concentration of aminemodified oligonucleotides. We estimate our total DNA density to be 6,500 DNA strands per particle by flow cytometry and control melting curve measurements, and use this value in all calculations presented. Hydrophobic dyes, either Nile red (MP Biomedicals) or BODIPY ${ }^{\circledR}$ 493/503 (Exciton), are incorporated easily into the polystyrene core by dissolving them in the toluene used for swelling.

\section{DNA sequence design}

All grafted sequences are 65-bases-long, single-stranded, and consist of an inert poly-dT spacer and a sticky end-containing, functional domain on the $3^{\prime}$ end. The poly-dT spacer sets the range of interaction; the sticky end sequence sets the strength and specificity of binding. All sequences are designed to minimize formation of stable secondary structures (such as hairpins) and crosstalk between non-interacting sequences by using three-base codons and their complements only once. Surfacegrafted strands are purified by high-performance liquid chromatography; short soluble strands are purified by standard desalting.

\section{Sample cell preparation and loading}

Sample chambers consist of two coverslips (No. 1; VWR) bonded together and sealed by silicone vacuum grease (Dow Corning) or UV-curable optical adhesive (Norland 63). The coverslips are plasma cleaned for approximately 1 minute to prevent nonspecific binding between DNA-grafted microspheres and the chamber walls. For melting curve measurements, $1.8-\mu \mathrm{m}$-diameter silica microspheres are added as spacers, so that DNA-grafted polystyrene particles form a colloidal monolayer, facilitating image analysis and determination of the singlet fraction.

\section{Melting curve measurements}

All melting curve measurements are done on quasi-two-dimensional suspensions. Samples are prepared by sealing a 1:1 binary mixture of particles at a total volume percent of $4 \%$ in a thin microscope chamber and imaged by an inverted optical microscope (Nikon TE2000-E). The height of the chamber is set to roughly $1.8 \mu \mathrm{m}$ by silica spacer particles. The sample temperature is controlled by a resistive objective heater coupled to the sample by immersion oil and a thermoelectric cooler 
(TE Technology, Inc.) bonded directly to the sample by silicone vacuum grease. The objective heater is driven by a low-noise temperature controller (Bioscience Tools); the thermoelectric cooler is driven by a separate high-performance digital temperature controller designed to drive thermoelectric cooler elements (Thorlabs). The sample is equilibrated at each temperature point for about $15 \mathrm{~min}$ before data is acquired. Each data point represents the average of three frames at a given temperature. Each frame is approximately $10^{4} \mu \mathrm{m}^{2}$ and contains about 1000 particles. The singlet fraction is determined using traditional image analysis routines (34). Our approach does not distinguish bound particles from those that are in close proximity but still unbound. We account for this systematic bias by comparing our measured singlet fraction to a simple Monte Carlo simulation of "hard disks" at the same concentration as our experiments. No noticeable hysteresis is observed for any of the melting curves.

\section{Modeling experimental data}

Model predictions are evaluated by fitting the model described in Section 9 to experimental measurements of the singlet fraction, and then comparing the fitted values of the enthalpy changes and entropy changes of hybridization to predictions from the nearest-neighbor model (NN model). Specifically, we evaluate the depth of the interparticle potential minimum according to Equation 1 and references $(7,24)$, and then use a separate model described in Refs. $(16,35)$ to relate the depth of the potential minimum to the singlet fraction.

\subsection{One-displacement scheme}

For the one-displacement scheme shown in Fig. 2, all data are fit simultaneously for the enthalpy changes and entropy changes of the two reactions depicted in Fig. 1B using nonlinear least squares regression. The fitted values of $\Delta H_{A B}, \Delta H_{A D_{1}}, \Delta S_{A B}$, and $\Delta S_{A D_{1}}$ are compared with predictions from the nearest-neighbor model (Table S2) and found to agree to within 3\%, well within the inherent uncertainty of roughly $8 \%$ in predictions of $\Delta H$ and $\Delta S(36)$. The same fitted values of the enthalpy and entropy change are used to compute all model curves shown in Fig. 2.

\subsection{Two-displacement scheme}

For the two-displacement scheme in Figs. 3-4, each melting curve at a given displacing strand concentration is fit independently. First, the enthalpy change of the bridge formation reaction $\Delta H_{A B}$ is fit to the melting curve measured in the absence of displacing strands $\left(C_{D 0}=0 \mu \mathrm{m}\right)$. The fitted value of $\Delta H_{A B}$ is again well within the inherent uncertainty of the nearest-neighbor model. This value of $\Delta H_{A B}$ and the $\mathrm{NN}$ prediction of $\Delta S_{A B}$ are fixed for calculations of the singlet fraction for suspensions with nonzero displacing strand concentrations.

Each melting curve measured at a finite displacing strand concentration is then fit for a single parameter $f_{\Delta H}$, where $f_{\Delta H}$ is a multiplicative factor that modifies the enthalpy change of the two displacement reactions according to $f_{\Delta H} \Delta H_{A D_{1}}$ and $f_{\Delta H} \Delta H_{B D_{2}}$. The entropy changes of the two displacement reactions are taken to be exactly the $\mathrm{NN}$ model predictions. We find that $f_{\Delta H}$ varies between 1.017 and 1.034 for all re-entrant melting curves, again well within the inherent uncertainty of the NN model, but exhibits a systematic trend, falling by roughly $2 \%$ as the temperature decreases from about $65{ }^{\circ} \mathrm{C}$ to $35^{\circ} \mathrm{C}$. We attribute this systematic deviation to the fact that the enthalpy change and entropy change upon hybridization are not strictly temperature-independent. In fact, nucleic acid hybridization and folding are also associated with changes in the heat capacity $\Delta C_{p}$. Assuming that $\Delta C_{p}$ is itself independent of temperature and the same for all hybridization reactions, 
we estimate that the change in heat capacity needed to produce a $2 \%$ systematic variation in $\Delta G$ of the displacement reactions over $30{ }^{\circ} \mathrm{C}$ is about $63 \mathrm{cal} /(\mathrm{mol} \mathrm{K})$ per base pair, consistent with experimental data (37). In fact we can compute a phase diagram in the $C_{D 0}-T$ space that reproduces our data (Fig. 3D) by fitting constant values of $\Delta H_{A B}, \Delta S_{A B}, \Delta H_{A D_{1}}, \Delta S_{A D_{1}}, \Delta H_{B D_{2}}$, and $\Delta S_{B D_{2}}$ (all within $8 \%$ of the NN predictions) and correcting for their temperature dependences using the estimated heat capacity $\Delta C_{p}$. We note that other experiments using DNA-grafted colloidal particles were likely insensitive to the temperature dependence of $\Delta H$ and $\Delta S$ due to the narrow temperature window of the aggregation-disaggregation transition for a single suspension.

\section{Image processing}

All three-color images from confocal fluorescence microscopy experiments are produced from twocolor images by the same algorithm. Emitted photons are collected simultaneously by two detectors. The signals from these detectors become red $r^{\prime}$ and blue $b^{\prime}$ channels. Red $r$, green $g$, and blue $b$ channels of the three-color images in Fig. 4B are computed according to

$$
\begin{aligned}
& r=r^{\prime}-\sqrt{r^{\prime} b^{\prime}} \\
& g=\sqrt{r^{\prime} b^{\prime}} \\
& b=b^{\prime}-\sqrt{r^{\prime} b^{\prime}}
\end{aligned}
$$

where the geometric mean $\sqrt{r^{\prime} b^{\prime}}$ is eroded and dilated once to minimize introduction of noise. These additional processing steps are performed only to improve clarity of the images and do not alter interpretation of the results.

\section{Colloidal crystallization}

All crystallization experiments are done using three-dimensional suspensions at a total volume percent of 5\%. Samples are mounted onto the stage of a resonant scanning confocal fluorescence microscope (Leica Microsystems) and incubated at constant temperature for roughly $2 \mathrm{hr}$. The temperature is controlled using the same heating elements and controllers as described above. After $2 \mathrm{hr}$, we find that more than $90 \%$ of the bound microspheres are incorporated into crystallites in coexistence with a dilute fluid. We estimate that the singlet fraction is roughly $0.1-0.3$. The colloid is nearly density matched by adding roughly $6 \%(\mathrm{w} / \mathrm{w})$ of sucrose to the buffer, minimizing sedimentation of the growing crystals. Samples are then imaged by confocal fluorescence microscopy. Particle species are identified by fluorescent dyes incorporated into their polystyrene cores: either Nile red, BODIPY ${ }^{\circledR} 493 / 503$, or a mixture of the two. All images are false-color.

\section{Crystal symmetry determination}

We identify the structure of our self-assembled binary crystals by comparing the measured lattice distances to known crystal structures of atomic solids. We estimate that greater than $90 \%$ of all crystals have the cesium chloride $(\mathrm{CsCl})$ symmetry, identified by two distinct features of the $\mathrm{CsCl}$ lattice (Fig. S3A-B): (1) the crystal planes are not close packed; and (2) the $\{110\}$ planes present distorted, isosceles triangles. Examples of $\{100\}$ planes are shown in Fig. S3C-D; examples of $\{110\}$ planes are shown in Fig. S3E-F. 


\section{Theoretical methods}

\subsection{Mean-field description of DNA-induced interactions}

We first seek to relate the thermodynamics of DNA-induced binding between colloidal particles to the thermodynamics of hybridization in solution, following an approach published previously $(6,7)$. The attractive free energy difference $\Delta F_{a}$ between a bound and unbound pair of particles is given by

$$
\frac{\Delta F_{a}}{k_{B} T}=-\ln \left(1+\frac{P_{\text {bound }}}{P_{\text {unbound }}}\right)=\ln P_{\text {unbound }},
$$

where $P_{\text {bound }}$ is the probabilities that the particles are bound together by DNA bridges, and $P_{\text {unbound }}$ is the probability that there are no bridges. To evaluate $P_{\text {unbound }}$ we make the simplifying assumption that chemical equilibrium is established locally. Note that we do not assume that the bridge distribution is Poissonian, an assumption that has been discussed previously in the literature $(7,24,38)$. Assuming local chemical equilibrium (LCE) only, $\Delta F_{a} / k_{B} T$ can be rewritten as

$$
\frac{\Delta F_{a}}{R T} \approx \int C_{i 0}(\mathbf{r}) \ln [1-\chi(\mathbf{r}, T)] \mathrm{d} \mathbf{r},
$$

where $C_{i 0}(\mathbf{r})$ is the limiting sticky-end concentration, $\chi(\mathbf{r}, T)$ the hybridization yield, $\mathbf{r}$ the spatial coordinate, and $T$ the temperature (24). The sticky-end concentrations can be generated numerically (7) by modeling the tethered DNA molecules as ideal chains of known contour and persistence length (39). The hybridization yield is calculated using the principles of mass-action, assuming that chemical equilibrium is established at each position $\mathbf{r}$. In the following sections, we derive a general expression for $\chi(T)$ that depends only on the concentration-adjusted free energy difference between bridging and non-bridging DNA configurations $\Delta G^{\prime}$, and we demonstrate how to manipulate $\Delta G^{\prime}$ by introducing competing reaction pathways.

\subsection{DNA hybridization as a bimolecular reaction}

Hybridization of complementary DNA sequences $A$ and $B$ can be modeled as the bimolecular reaction (40)

$$
A+B \leftrightharpoons A B
$$

We assume the starting concentrations of $A$ and $B$ are $C_{A 0}$ and $C_{B 0}=b C_{A 0}$, where $b \geq 1$. The hybridization yield $\chi=C_{A B} /\left(C_{A}+C_{A B}\right)=C_{A B} / C_{A 0}$ can be evaluated by solving the system of equations

$$
\begin{aligned}
K_{e q}(T) & =\frac{C_{A B} C^{\circ}}{C_{A} C_{B}} \\
C_{A 0} & =C_{A}+C_{A B} \\
C_{B 0} & =b C_{A 0}=C_{B}+C_{A B},
\end{aligned}
$$

where $C_{i}$ is the concentration of species $i$ in equilibrium, $C^{\circ}=1 \mathrm{M}$ is a reference concentration, $K_{e q}(T)=\exp \left[-\beta \Delta G_{A B}(T)\right]$ is the equilibrium constant, $1 / \beta$ is the thermal energy $k_{B} T$, and $\Delta G_{A B}=\Delta H_{A B}-T \Delta S_{A B}$ is the standard free energy difference between double-stranded $A B$ and single-stranded $A$ and $B$ at the reference concentration $C^{\circ}$. The resulting hybridization yield,

$$
\chi=\frac{(b+1) K_{e q} \frac{C_{A 0}}{C^{\circ}}+1-\sqrt{(b-1)^{2}\left(K_{e q} \frac{C_{A 0}}{C^{\circ}}\right)^{2}+2(b+1) K_{e q} \frac{C_{A 0}}{C^{\circ}}+1}}{2 K_{e q} \frac{C_{A 0}}{C^{\circ}}},
$$


depends only on the concentration-adjusted equilibrium constant $K_{e q}^{\prime}=K_{e q} C_{A 0} / C^{\circ}$. The concentrationadjusted standard free energy $\Delta G^{\prime} \equiv-R T \ln K_{e q}^{\prime}$ is then

$$
\frac{\Delta G^{\prime}}{R T}=\frac{\Delta H_{A B}}{R T}-\left[\frac{\Delta S_{A B}}{R}+\ln \left(\frac{C_{A 0}}{C^{\circ}}\right)\right] .
$$

The additional entropic term $R \ln \left(C_{A 0} / C^{\circ}\right)$ results from the fact that two single-stranded reactants are consumed upon formation of one double-stranded product. The temperature dependence of Equation 3, and thus of Equation 1, is determined entirely by $\Delta H_{A B}$.

Equations 1, 2, and 3 relate the thermodynamics of hybridization of DNA in solution $\Delta G^{\prime}$ to the thermodynamics of DNA-induced binding between particles $\Delta F_{a}$, with the caveat that both $b$ and $C_{A 0}$ are spatially varying. In what follows, we will show that bridging in the presence of competing hybridization reactions can be modeled by Equations 1 and 2, and that the competing interactions serve only to modify the expression of $\Delta G^{\prime}$. The results presented above are independent of our choice of $b \geq 1$; if $b<1$ the hybridization yield is $\chi=C_{A B} /\left(C_{B}+C_{A B}\right)=C_{A B} /\left(b C_{A 0}\right)$, and $C_{i 0}(\mathbf{r})$ in Equation 1 becomes $b(\mathbf{r}) C_{A 0}(\mathbf{r})$, but otherwise the equations remain unchanged. We assume $b \geq 1$ for everything that follows.

\subsection{Controlling $\Delta G^{\prime}$ through a single toehold-exchange reaction}

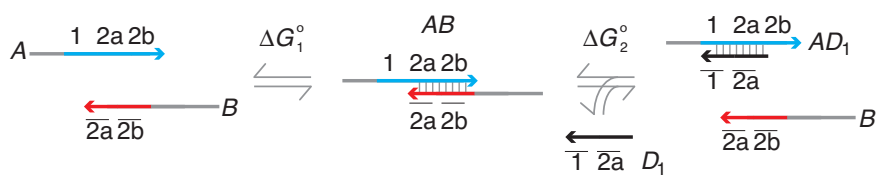

Fig. SM1. A single displacement reaction. Strands $A$ and $B$ are grafted to the surface of colloidal microspheres (not shown); strand $D_{1}$ is free in solution. The DNA strands are conceptually divided into functional domains whose sequences determine the interactions between the various species. The domains are grouped by purpose. Domains 1 and $2 \mathrm{~b}$ are termed toeholds; domains 2 and $\overline{2}$ are the "sticky ends". The sticky ends hybridize to form double-stranded DNA bridges; the toeholds accelerate the initiation of strand-displacement reactions that form or rupture these bridges by a process called toehold exchange. The free energy differences $\Delta G_{1}^{\circ}$ and $\Delta G_{2}^{\circ}$ are determined by the sequences of $A, B$, and $D_{1}$.

The chemical reactions describing the competition between a single strand-displacement reaction (Fig. SM1) and bridge formation are

$$
\begin{aligned}
A+B & \leftrightharpoons A B \\
A B+D_{1} & \leftrightharpoons A D_{1}+B
\end{aligned}
$$

where $D_{1}$ is a soluble strand that is complementary to a portion of strand $A$. The hybridization yield $\chi(T)$ can be evaluated by solving the system of equations

$$
\begin{aligned}
K_{e q}^{(1)}(T) & =\frac{C_{A B} C^{\circ}}{C_{A} C_{B}} \\
K_{e q}^{(2)}(T) & =\frac{C_{A D_{1}} C_{B}}{C_{A B} C_{D_{1}}} \\
C_{A 0} & =C_{A}+C_{A B}+C_{A D_{1}} \\
C_{B 0} & =b C_{A 0}=C_{B}+C_{A B} \\
C_{D_{1} 0} & \approx C_{D_{1}},
\end{aligned}
$$


where we assume that the soluble strand $D_{1}$ is not consumed by the reaction, an assumption that is valid if $D_{1}$ is in large molecular excess. Because the grafted DNA strands are confined roughly within a spherical shell with volume $V_{\text {shell }} \approx 4 \pi a^{2} L$, this condition is satisfied so long as $C_{D_{1} 0} / C_{A 0} \gg$ $\phi /(1-\phi) \cdot V_{\text {shell }} / V_{\text {particle }}=3 \phi /(1-\phi) \cdot L / a$, where $\phi$ is the colloid volume fraction, $a$ is the particle radius, and $L \approx 15 \mathrm{~nm}$ is the thickness of the DNA brush. For our experimental conditions, $3 \phi /(1-\phi) \cdot L / a \approx 0.005$.

Assuming the starting concentrations of $A, B$, and $D_{1}$ are $C_{A 0}, C_{B 0}=b C_{A 0}$, and $C_{D_{1} 0}$, the hybridization yield $\chi=C_{A B} /\left(C_{A}+C_{A B}+C_{A D_{1}}\right)=C_{A B} / C_{A 0}$ can be expressed in the same form as Equation 2,

$$
\chi=\frac{(b+1) K_{e q}^{\prime}+1-\sqrt{(b-1)^{2}\left(K_{e q}^{\prime}\right)^{2}+2(b+1) K_{e q}^{\prime}+1}}{2 K_{e q}^{\prime}},
$$

but where the concentration-adjusted standard free energy is now given by

$$
\frac{\Delta G^{\prime}}{R T}=-\ln \left[\frac{K_{e q}^{(1)} C_{A 0} / C^{\circ}}{1+K_{e q}^{(1)} K_{e q}^{(2)} C_{D_{1} 0} / C^{\circ}}\right] .
$$

Thus the net effect of the competing toehold-exchange reaction is to modify the concentrationadjusted standard free energy $\Delta G^{\prime}$.

While we can no longer extract a simple, compact expression for $\Delta G^{\prime}$ for all temperatures, it is helpful to consider what happens in different limits. In the high-temperature limit, where $K_{e q}^{(1)} K_{e q}^{(2)} C_{D_{1} 0} / C^{\circ} \ll 1, \Delta G^{\prime}$ reduces to

$$
\frac{\Delta G_{\ll 1}^{\prime}}{R T}=\frac{\Delta H_{A B}}{R T}-\left[\frac{\Delta S_{A B}}{R}+\ln \left(\frac{C_{A 0}}{C^{\circ}}\right)\right]
$$

the same as the free energy difference in the absence of strand $D_{1}$ (Equation 3). In the opposite, low-temperature limit

$$
\frac{\Delta G_{\gg 1}^{\prime}}{R T}=\frac{\left(\Delta H_{A B}-\Delta H_{A D_{1}}\right)}{R T}-\left[\frac{\left(\Delta S_{A B}-\Delta S_{A D_{1}}\right)}{R}-\ln \left(\frac{C_{D_{1} 0}}{C_{A 0}}\right)\right] .
$$

Here, we find that the displacement reaction acts to effectively lower the free energy difference between the bridging and non-bridging configurations by an amount equal to the thermodynamic stability of the $A D_{1}$ duplex. As a result, for some strands $D_{1}$ and $B$ that undergo the same enthalpy change upon hybridization to $A$ (that is, $\Delta H_{A B}=\Delta H_{A D_{1}}$ ),

$$
\frac{\Delta G_{\gg 1}^{\prime}}{R T}=-\left[\frac{\left(\Delta S_{A B}-\Delta S_{A D_{1}}\right)}{R}-\ln \left(\frac{C_{D_{1} 0}}{C_{A 0}}\right)\right]
$$

becomes independent of temperature. Under these conditions, $\Delta G^{\prime} / R T$ is determined entirely by entropy. Note that $\Delta G^{\prime}$ and thus the pair-interaction potential can still be adjusted by changing the displacing strand concentration $C_{D_{1} 0}$. As we show in the main text, this type of interaction is useful for softening or even eliminating the temperature dependence of binding between DNAgrafted particles. The full temperature dependence of $\Delta G^{\prime}$ for different relative displacing strand concentrations $C_{D_{1} 0} / C_{A 0}$ is shown in Fig. $\mathrm{S} 1$. 


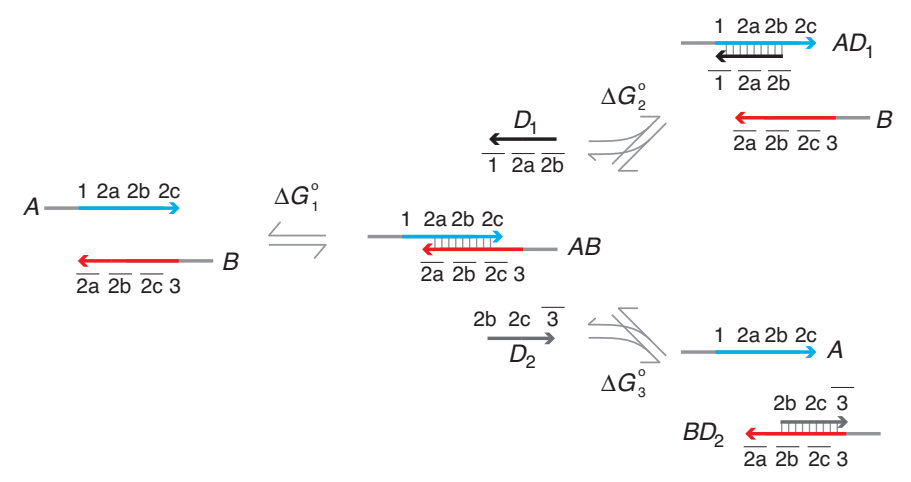

Fig. SM2. Two competing displacement reactions. Strands $A$ and $B$ are grafted to the surface of colloidal microspheres (not shown); strand $D_{1}$ and $D_{2}$ are free in solution. Domains $1,2 \mathrm{a}, 2 \mathrm{c}$, and 3 are toeholds; domains 2 and $\overline{2}$ are the sticky ends. Domains $2 \mathrm{~b}$ and $2 \overline{\mathrm{b}}$ destabilize the four-strand motif $A B D_{1} D_{2}$ in favor of the two duplexes $A D_{1}$ and $B D_{2}$.

\subsection{Two toehold-exchange reactions induce re-entrant melting}

Addition of two soluble displacing strands $D_{1}$ and $D_{2}$ (Fig. SM2) that compete with bridge formation according to

$$
\begin{aligned}
A+B & \leftrightharpoons A B \\
A B+D_{1} & \leftrightharpoons A D_{1}+B \\
A B+D_{2} & \leftrightharpoons A+B D_{2}
\end{aligned}
$$

further modifies the concentration-adjusted standard free energy difference $\Delta G^{\prime}$. We derive the concentration-adjusted standard free energy $\Delta G^{\prime}$ by solving the system of equations

$$
\begin{aligned}
K_{e q}^{(1)} & =\frac{C_{A B} C^{\circ}}{C_{A} C_{B}} \\
K_{e q}^{(2)} & =\frac{C_{A D_{1}} C_{B}}{C_{A B} C_{D_{1}}} \\
K_{e q}^{(3)} & =\frac{C_{B D_{2}} C_{A}}{C_{A B} C_{D_{2}}} \\
C_{A 0} & =C_{A}+C_{A B}+C_{A D_{1}} \\
C_{B 0} & =b C_{A 0}=C_{B}+C_{A B}+C_{B D_{2}} \\
C_{D_{1} 0} & \approx C_{D_{1}} \\
C_{D_{2} 0} & \approx C_{D_{2}}
\end{aligned}
$$

for the hybridization yield $\chi=C_{A B} /\left(C_{A}+C_{A B}+C_{A D_{1}}\right)$, then rearranging $\chi$ to match Equation 4 . The expression that we find,

$$
\frac{\Delta G^{\prime}}{R T}=-\ln \left[\frac{K_{e q}^{(1)} C_{A 0} / C^{\circ}}{\left[1+K_{e q}^{(1)} K_{e q}^{(2)} C_{D_{1} 0} / C^{\circ}\right]\left[1+K_{e q}^{(1)} K_{e q}^{(3)} C_{D_{2} 0} / C^{\circ}\right]}\right],
$$

contains an additional factor in the denominator when compared to Equation 5, and reduces to Equation 5 exactly when $C_{D_{2} 0}=0$. 
For equimolar mixtures of the two displacing strands $\left(C_{D_{1} 0}=C_{D_{2} 0}=C_{D 0}\right)$, we again evaluate the concentration-adjusted standard free energy difference between the bridging and non-bridging conformations in different limits. For example, when the three duplexes are thermodynamically equivalent (that is, $\Delta G_{A B}=\Delta G_{A D_{1}}=\Delta G_{B D_{2}}$ ), $\Delta G^{\prime}$ again reduces to

$$
\frac{\Delta G_{\ll 1}^{\prime}}{R T}=\frac{\Delta H_{A B}}{R T}-\left[\frac{\Delta S_{A B}}{R}+\ln \left(\frac{C_{A 0}}{C^{\circ}}\right)\right]
$$

in the limit where $K_{e q}^{(1)} K_{e q}^{(2)} C_{D 0} / C^{\circ} \ll 1$. In the opposite, low-temperature limit,

$$
\frac{\Delta G_{\gg 1}^{\prime}}{R T}=-\frac{\Delta H_{A B}}{R T}+\left[\frac{\Delta S_{A B}}{R}+\ln \left(\frac{C_{A 0}}{C^{\circ}}\right)+2 \ln \left(\frac{C_{D 0}}{C_{A 0}}\right)\right] .
$$

Under these conditions, we find that $\Delta G_{\gg 1}^{\prime} / R T$ has an inverted temperature dependence compared to Equation 3. In other words, the concentration-adjusted standard free energy difference between bridging and non-bridging conformations actually becomes larger as the temperature decreases, making bridge formation less thermodynamically favorable. This inversion in the temperature dependence can be used to induce two melting transitions: one upon heating (driven by entropic gain) and another upon cooling (driven by enthalpic gain) as shown in the main text. The location and steepness of the re-entrant melting transition can be adjusted by changing the relative displacing strand concentration and the enthalpy change of displacement, respectively. The full temperature dependence of $\Delta G^{\prime}$ is plotted in Fig. S2. 


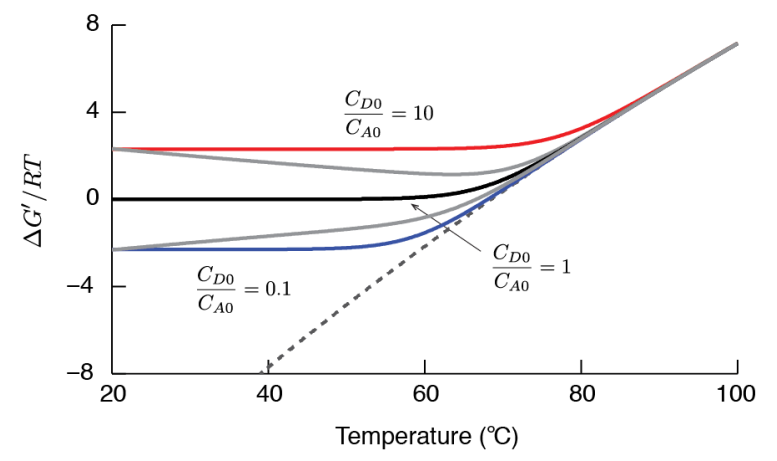

Fig. S1. A single toehold-exchange reaction eliminates the temperature dependence of the concentration-adjusted standard free energy difference. The asymptote at high temperature is given by $\Delta G_{\ll 1}^{\prime} / R T$; the asymptote at low temperature is given by $\Delta G_{\gg 1}^{\prime} / R T$. The low-temperature asymptotic value of $\Delta G^{\prime} / R T$ can be adjusted by changing the relative displacing strand concentration $C_{D_{1} 0} / C_{A 0}$ (red, black, and blue curves). The temperature dependence of $\Delta G_{\gg 1}^{\prime} / R T$ can be adjusted by changing $\Delta H_{A D_{1}}$ relative to $\Delta H_{A B}$ (gray curves). The displacement-free case (dashed curve) is recovered when $C_{D_{1} 0}=0$. Red, black, and blue curves correspond to Equation 5 for $\Delta H_{A B}=\Delta H_{A D_{1}}=-57.5 \mathrm{kcal} / \mathrm{mol}, \Delta S_{A B}=\Delta S_{A D_{1}}=-150 \mathrm{cal} / \mathrm{mol} \cdot \mathrm{K}$, and $C_{A 0}=100 \mu \mathrm{M}$. Gray curves are for $\Delta H_{A D_{1}}=(1 \pm 0.1) \Delta H_{A B}$ and $\Delta S_{A D_{1}}=(1 \pm 0.1) \Delta S_{A B}$. 


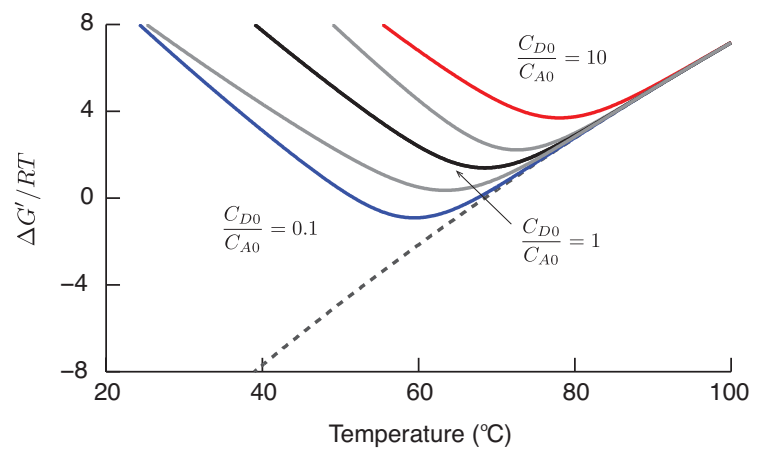

Fig. S2. Two displacement reactions induce a non-monotonic temperature dependence in the concentration-adjusted standard free energy difference. The asymptote at high temperature is given by $\Delta G_{\ll 1}^{\prime}$; the asymptote at low temperature is given by $\Delta G_{\gg 1}^{\prime}$. The low-temperature asymptotic value of $\Delta G^{\prime} / R T$ can be adjusted by changing the relative displacing strand concentration $C_{D 0} / C_{A 0}$, for equimolar mixtures of $D_{1}$ and $D_{2}$ (red, black, and blue curves). The temperature dependence of $\Delta G_{\gg 1}^{\prime} / R T$ can be adjusted by changing $\Delta H_{A D_{1}}$ and $\Delta H_{B D_{2}}$ relative to $\Delta H_{A B}$ (gray curves). The displacement-free case (dashed curve) is recovered when $C_{D 0}=0$. Red, black, and blue curves correspond to Equation 7 for $\Delta H_{A B}=\Delta H_{A D_{1}}=\Delta H_{B D_{2}}=-57.5 \mathrm{kcal} / \mathrm{mol}, \Delta S_{A B}=\Delta S_{A D_{1}}=\Delta S_{B D_{2}}=$ $-150 \mathrm{cal} / \mathrm{mol} \cdot \mathrm{K}$, and $C_{A 0}=100 \mu \mathrm{M}$. Gray curves are for $\Delta H_{A D_{1}}=\Delta H_{B D_{2}}=(1 \pm 0.1) \Delta H_{A B}$ and $\Delta S_{A D_{1}}=\Delta S_{B D_{2}}=(1 \pm 0.1) \Delta S_{A B}$. 

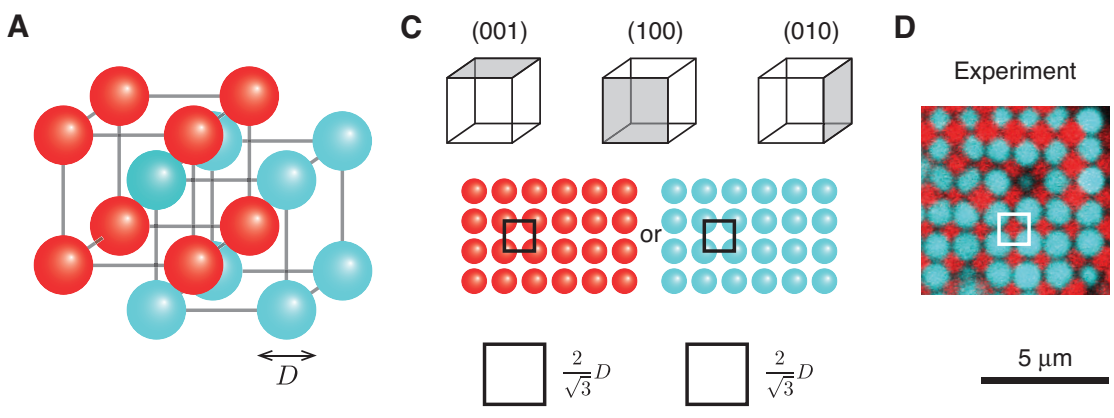

$5 \mu \mathrm{m}$

B

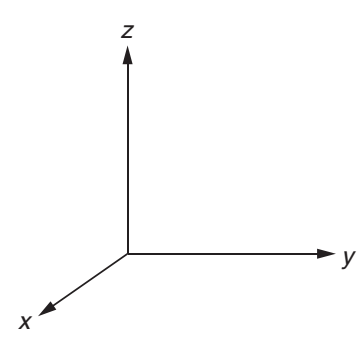

$\mathrm{E}$<smiles>OC12C3C4C5C3C1C5C42</smiles>
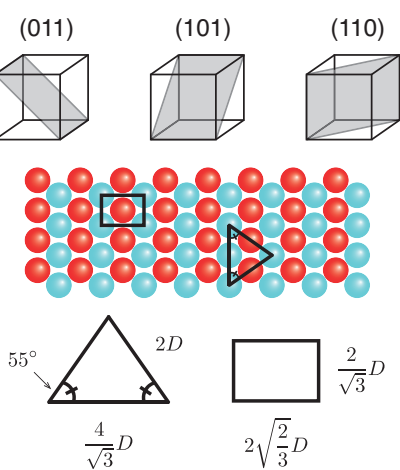

(110) $\mathbf{F}$

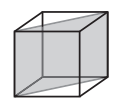

Experiment

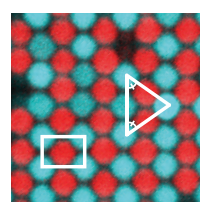

$5 \mu \mathrm{m}$

Fig. S3. Crystallography of the cesium chloride $(\mathrm{CsCl})$ binary crystals. (A) The $\mathrm{CsCl}$ structure consists of two interpenetrating cubic lattices, where both particle types have eightfold coordination. For the coordinate system in $(\mathbf{B}),(\mathbf{C})$ shows representative $\{100\}$ planes and an experimental example (D); (E) shows representative $\{110\}$ planes and an experimental example $(\mathbf{F})$. The lattice distances are given as a function of the particle diameter $D$. The distorted triangles in $(\mathbf{E}-\mathbf{F})$ are a hallmark of the $\{110\}$ planes of the $\mathrm{CsCl}$ lattice. 
Table S1. DNA sequences and buffer conditions for temperature-independent binding experiments. Sequences $A$ and $B$ are grafted to colloidal microspheres; sequence $D_{1}$ is free in solution. Experiments were performed in aqueous buffer containing $11 \mathrm{mM} \mathrm{MgCl} 2,10 \mathrm{mM}$ Tris, $1 \mathrm{mM}$ EDTA.

\begin{tabular}{cc} 
Strand name & Base sequence \\
\hline$A$ & $5^{\prime}-(\mathrm{T})_{51}-$ GAGTTGCGGTAGAC-3' \\
$B$ & $5^{\prime}-(\mathrm{T})_{51}$-AATGCCTGTCTACC-3' \\
$D_{1}$ & 5'-ACCGCAA-3'
\end{tabular}

Table S2. Thermodynamic parameters for temperature-independent binding experiments. The standard enthalpy change and entropy change of hybridization for $C^{\circ}=1 \mathrm{M}$ are calculated using NUPACK nucleic acid package for $50 \mathrm{mM} \mathrm{NaCl}, 10 \mathrm{mM} \mathrm{MgCl}_{2}$, and "some" dangle treatment (32). Although our experiments were performed in the absence of $\mathrm{NaCl}$, NUPACK requires a minimum of $50 \mathrm{mM} \mathrm{NaCl}$. We do not expect this contribution to the predicted free energies to be significant. We only use $10 \mathrm{mM} \mathrm{MgCl}_{2}$ in our calculation since $1 \mathrm{mM}$ of the $11 \mathrm{mM}$ is chelated by $1 \mathrm{mM}$ EDTA.

\begin{tabular}{ccc} 
Duplex name & $\Delta H(\mathrm{kcal} / \mathrm{mol})$ & $\Delta S(\mathrm{cal} / \mathrm{mol} / \mathrm{K})$ \\
\hline$A B$ & -56.6 & -163.0 \\
$A D_{1}$ & -53.6 & -149.1
\end{tabular}


Table S3. DNA sequences and buffer conditions for re-entrant melting experiments. Sequences $A$ and $B$ are grafted to colloidal microspheres; sequences $D_{1}$ and $D_{2}$ are free in solution. Experiments were performed in aqueous buffer containing $250 \mathrm{mM} \mathrm{NaCl}, 10 \mathrm{mM}$ Tris, $1 \mathrm{mM}$ EDTA.

\begin{tabular}{cc} 
Strand name & Base sequence \\
\hline$A$ & 5'-(T) $_{51}$-CTAACTGCGGT-3' \\
$B$ & ${\text { 5'-(T) }{ }_{51} \text {-CTTACCGCAGT-3' }}^{\prime}$ '-GCAGTTAG-3' \\
$D_{1}$ & 5'-GCGGTAAG-3'
\end{tabular}

Table S4. Thermodynamic parameters for re-entrant melting experiments. The standard enthalpy change and entropy change of hybridization for $C^{\circ}=1 \mathrm{M}$ are calculated using NUPACK nucleic acid package for $250 \mathrm{mM} \mathrm{NaCl}$ and "some" dangle treatment (32).

\begin{tabular}{ccc} 
Duplex name & $\Delta H(\mathrm{kcal} / \mathrm{mol})$ & $\Delta S(\mathrm{cal} / \mathrm{mol} / \mathrm{K})$ \\
\hline$A B$ & -63.6 & -173.7 \\
$A D_{1}$ & -64.4 & -186.1 \\
$B D_{2}$ & -69.4 & -195.7
\end{tabular}


Table S5. DNA sequences and buffer conditions for "reconfigurable" self-assembly of a binary crystal. Sequences $A, B, E$, and $F$ are grafted to colloidal microspheres; sequences $D_{1}$ and $D_{2}$ are free in solution. The Red and Blue particles each have a single sequence grafted to their surfaces; the Green particle has a 1:1 mixture of two different sequences grafted to its surface. Experiments were performed in aqueous buffer containing $500 \mathrm{mM} \mathrm{NaCl}, 10 \mathrm{mM}$ Tris, $1 \mathrm{mM}$ EDTA.

\begin{tabular}{|c|c|c|}
\hline Particle type & Strand name & Base sequence \\
\hline Red & $A$ & $5^{\prime}-(\mathrm{T})_{51}$-CTAACTGCGGT-3' \\
\hline Green & $B$ & $5^{\prime}-(\mathrm{T})_{51}$-CTTACCGCAGT-3' \\
\hline Green & E & $5^{\prime}-(\mathrm{T})_{59}-\mathrm{CAGGTG}-3^{\prime}$ \\
\hline Blue & $F$ & $5^{\prime}-(\mathrm{T})_{59}-\mathrm{CACCTG}-3^{\prime}$ \\
\hline - & $D_{1}$ & 5'-GCAGTTAG-3' \\
\hline - & $D_{2}$ & 5'-GCGGTAAG-3' \\
\hline
\end{tabular}

Table S6. Thermodynamic parameters for 'reconfigurable' self-assembly of a binary crystal. The standard enthalpy change and entropy change of hybridization for $C^{\circ}=1 \mathrm{M}$ are calculated using NUPACK nucleic acid package for $500 \mathrm{mM} \mathrm{NaCl}$ and "some" dangle treatment (32).

\begin{tabular}{ccc} 
Duplex name & $\Delta H(\mathrm{kcal} / \mathrm{mol})$ & $\Delta S(\mathrm{cal} / \mathrm{mol} / \mathrm{K})$ \\
\hline$A B$ & -63.6 & -171.5 \\
$A D_{1}$ & -64.6 & -184.8 \\
$B D_{2}$ & -69.6 & -194.1 \\
$E F$ & -49.0 & -138.0
\end{tabular}




\section{References}

1. D. Frenkel, Playing tricks with designer "atoms", Science 296, 65 (2002).

2. C. A. Mirkin, R. L. Letsinger, R. C. Mucic, J. J. Storhoff, A DNA-based method for rationally assembling nanoparticles into macroscopic materials, Nature 382, 607 (1996).

3. A. P. Alivisatos, K. P. Johnsson, X. Peng, T. E. Wilson, C. J. Loweth, M. P. Bruchez, P. G. Schultz, Organization of 'nanocrystal molecules' using DNA, Nature 382, 609 (1996).

4. R. J. Macfarlane, M. N. O’Brien, S. H. Petrosko, C. A. Mirkin, Nucleic acid-modified nanostructures as programmable atom equivalents: Forging a new "table of elements", Angew. Chem. Int. Ed. 52, 5688 (2013).

5. R. Macfarlane, B. Lee, M. Jones, N. Harris, G. Schatz, C. Mirkin, Nanoparticle superlattice engineering with DNA, Science 334, 204 (2011).

6. P. L. Biancaniello, A. J. Kim, J. C. Crocker, Colloidal interactions and self-assembly using DNA hybridization, Phys. Rev. Lett. 94, 058302 (2005).

7. W. B. Rogers, J. C. Crocker, Direct measurements of DNA-mediated colloidal interactions and their quantitative modeling, Proc. Natl Acad. Sci. USA 108, 15687 (2011).

8. P. Varilly, S. Angioletti-Uberti, B. Mognetti, D. Frenkel, A general theory of DNA-mediated and other valence-limited colloidal interactions, J. Chem. Phys. 137, 094108 (2012).

9. J. SantaLucia, A unified view of polymer, dumbbell, and oligonucleotide DNA nearestneighbor thermodynamics, Proc. Natl Acad. Sci. USA 95, 1460 (1998).

10. D. Nykypanchuk, M. Maye, D. van der Lelie, O. Gang, DNA-guided crystallization of colloidal nanoparticles, Nature 451, 549 (2008).

11. S. Park, A. Lytton-Jean, B. Lee, S. Weigand, G. Schatz, C. Mirkin, DNA-programmable nanoparticle crystallization, Nature 451, 553 (2008).

12. R. J. Macfarlane, M. R. Jones, B. Lee, E. Auyeung, C. A. Mirkin, Topotactic interconversion of nanoparticle superlattices, Science 341, 1222 (2013).

13. M. Casey, R. Scarlett, W. Rogers, I. Jenkins, T. Sinno, J. Crocker, Driving diffusionless transformations in colloidal crystals using DNA handshaking, Nat. Commun. 3, 1209 (2012).

14. J. D. Halverson, A. V. Tkachenko, DNA-programmed mesoscopic architecture, Phys. Rev. E 87, 062310 (2013).

15. Z. Zeravcic, V. N. Manoharan, M. P. Brenner, Size limits of self-assembled colloidal structures made using specific interactions, Proc. Natl Acad. Sci. USA 111, 15918 (2014).

16. R. Dreyfus, M. E. Leunissen, R. Sha, A. V. Tkachenko, N. C. Seeman, D. J. Pine, P. M. Chaikin, Simple quantitative model for the reversible association of DNA coated colloids, Phys. Rev. Lett. 102, 048301 (2009).

17. L. Di Michele, E. Eiser, Developments in understanding and controlling self assembly of DNAfunctionalized colloids, Phys. Chem. Chem. Phys. 15, 3115 (2013).

18. B. Yurke, A. J. Turberfield, A. P. Mills, F. C. Simmel, J. L. Neumann, A DNA-fuelled molecular machine made of DNA, Nature 406, 605 (2000).

19. D. Y. Zhang, G. Seelig, Dynamic DNA nanotechnology using strand-displacement reactions, Nature Chem. 3, 103 (2011).

20. C. K. Tison, V. T. Milam, Reversing DNA-mediated adhesion at a fixed temperature, Langmuir 23, 9728 (2007).

21. M. Maye, M. Kumara, D. Nykypanchuk, W. Sherman, O. Gang, Switching binary states of nanoparticle superlattices and dimer clusters by DNA strands, Nature Nanotech. 5, 116 (2010). 
22. J. T. McGinley, I. Jenkins, T. Sinno, J. C. Crocker, Assembling colloidal clusters using crystalline templates and reprogrammable DNA interactions, Soft Matter 9, 9119 (2013).

23. B. A. Baker, G. Mahmoudabadi, V. T. Milam, Strand displacement in DNA-based materials systems, Soft Matter 9, 11160 (2013).

24. W. B. Rogers, J. C. Crocker, Reply to Mognetti et al.: DNA handshaking interaction data are well described by mean-field and molecular models, Proc. Natl Acad. Sci. USA 109, E380 (2012).

25. Materials and methods are available as supplementary material on Science online.

26. T. I. Li, R. Sknepnek, M. Olvera de la Cruz, Thermally active hybridization drives the crystallization of DNA-functionalized nanoparticles, J. Am. Chem. Soc. 135, 8535 (2013).

27. R. Scarlett, M. Ung, J. Crocker, T. Sinno, A mechanistic view of binary colloidal superlattice formation using DNA-directed interactions, Soft Matter 7, 1912 (2011).

28. S. Angioletti-Uberti, B. M. Mognetti, D. Frenkel, Re-entrant melting as a design principle for DNA-coated colloids, Nature Mater. 11, 518 (2012).

29. B. Mognetti, M. Leunissen, D. Frenkel, Controlling the temperature sensitivity of DNAmediated colloidal interactions through competing linkages, Soft Matter 8, 2213 (2012).

30. S. Roldan-Vargas, F. Smallenburg, W. Kob, F. Sciortino, Gelling by heating, Sci. Rep. 3, 2451 (2013).

31. J. King, S. Casjens, Catalytic head assembling protein in virus morphogenesis, Nature 251, 112 (1974).

32. J. N. Zadeh, C. D. Steenberg, J. S. Bois, B. R. Wolfe, M. B. Pierce, A. R. Khan, R. M. Dirks, N. A. Pierce, Nupack: analysis and design of nucleic acid systems, J. Comput. Chem. 32, 170 (2011).

33. A. Kim, V. Manoharan, J. Crocker, Swelling-based method for preparing stable, functionalized polymer colloids, J. Am. Chem. Soc. 127, 1592 (2005).

34. J. Crocker, D. Grier, et al., Methods of digital video microscopy for colloidal studies, J. Colloid Interface Sci. 179, 298 (1996).

35. K. Wu, L. Feng, R. Sha, R. Dreyfus, A. Grosberg, N. Seeman, P. Chaikin, Polygamous particles, Proceedings of the National Academy of Sciences 109, 18731 (2012).

36. J. SantaLucia, H. T. Allawi, P. A. Seneviratne, Improved nearest-neighbor parameters for predicting DNA duplex stability, Biochemistry 35, 3555 (1996).

37. J. SantaLucia Jr, The use of spectroscopic techniques in the study of DNA stability, Spectrophotometry and Spectrofluorimetry: A Practical Approach pp. 329-56 (2000).

38. B. M. Mognetti, P. Varilly, S. Angioletti-Uberti, F. J. Martinez-Veracoechea, J. Dobnikar, M. E. Leunissen, D. Frenkel, Predicting DNA-mediated colloidal pair interactions, Proc. Natl Acad. Sci. USA 109, E378 (2012).

39. M. Murphy, I. Rasnik, W. Cheng, T. M. Lohman, T. Ha, Probing single-stranded DNA conformational flexibility using fluorescence spectroscopy, Biophys. J. 86, 2530 (2004).

40. D. Y. Zhang, S. X. Chen, P. Yin, Optimizing the specificity of nucleic acid hybridization, Nature Chem. 4, 208 (2012). 